\title{
OPEN Oncogenic G12D mutation alters local conformations and dynamics of K-Ras
}

Received: 6 November 2018

Accepted: 29 July 2019

Published online: 13 August 2019

\begin{abstract}
Sezen Vatansever ${ }^{1,2}$, Burak Erman ${ }^{3}$ \& Zeynep H. Gümüş $\mathbb{1 0}^{1,2}$
K-Ras is the most frequently mutated oncoprotein in human cancers, and G12D is its most prevalent mutation. To understand how G12D mutation impacts K-Ras function, we need to understand how it alters the regulation of its dynamics. Here, we present local changes in K-Ras structure, conformation and dynamics upon G12D mutation, from long-timescale Molecular Dynamics simulations of active (GTP-bound) and inactive (GDP-bound) forms of wild-type and mutant K-Ras, with an integrated investigation of atomistic-level changes, local conformational shifts and correlated residue motions. Our results reveal that the local changes in K-Ras are specific to bound nucleotide (GTP or GDP), and we provide a structural basis for this. Specifically, we show that G12D mutation causes a shift in the population of local conformational states of K-Ras, especially in Switch-II (SII) and $\alpha 3$-helix regions, in favor of a conformation that is associated with a catalytically impaired state through structural changes; it also causes SII motions to anti-correlate with other regions. This detailed picture of G12D mutation effects on the local dynamic characteristics of both active and inactive protein helps enhance our understanding of local K-Ras dynamics, and can inform studies on the development of direct inhibitors towards the treatment of K-Ras ${ }^{\mathrm{G} 12 \mathrm{D}}$-driven cancers.
\end{abstract}

$\mathrm{K}-\mathrm{Ras}$ is the most frequently mutated oncoprotein in multiple human cancers ${ }^{1-3}$. Patients with oncogenic K-Ras mutations have very poor response to standard therapies. Unfortunately, these mutations eventually emerge during the course of their treatment and drive resistance ${ }^{4-6}$. The importance of oncogenic mutations in K-Ras stem from its pivotal role in signaling networks that control cellular growth, proliferation, and differentiation ${ }^{7}$. To perform its cellular roles, K-Ras continuously switches between GDP-bound (inactive) and GTP-bound (active) states (Fig. 1A) ${ }^{8,9}$. This switch mechanism is important in turning signals through K-Ras on or off, because only active $\mathrm{K}$-Ras can bind to and trigger its downstream proteins ${ }^{10}$. During the switch, active K-Ras (K-Ras-GTP) acts as a GTPase by hydrolyzing GTP and becomes inactive (K-Ras-GDP). K-Ras-GTP may also bind to GTPase-activating proteins (GAPs) that accelerate this process ${ }^{11,12}$. However, oncogenic K-Ras mutations impair both its GTPase activity and GAP protein binding, inhibiting GTP hydrolysis. Thus, unable to switch to its GDP-bound (inactive) state, mutant K-Ras remains continuously active, leading to prolonged activation of its downstream pathways associated with oncogenic cellular growth ${ }^{10,13-15}$. There is strong evidence that blocking mutant K-Ras activity can be very effective in treatment ${ }^{16,17}$. Yet, despite decades of research, there are still no drugs in the clinic today that directly target K-Ras mutants ${ }^{18,19}$.

Activating oncogenic K-Ras mutations are frequently observed at residue positions 12, 13 and 61 in cancer patients. Among these, G12 is the most frequently mutated residue (89\%) and it most often mutates to aspartate (G12D, 36\%) followed by valine (G12V, 23\%) and cysteine (G12C, 14\%) $)^{3,10}$. Therefore, in this study we focus on the most frequently observed K-Ras mutation in cancer patients, G12D. G12 is located at the protein active site, which consists of a phosphate binding loop (P-loop, residues 10-17) and two switch regions (Switch-I (SI), residues 25-40, and Switch-II (SII), residues 60-74) (Fig. 1B, C). The residues in the active site bind to the phosphate groups of GTP and are responsible for the GTPase function of K-Ras. The switch regions SI and SII are additionally responsible for controlling binding to effector and regulator proteins (Fig. 1D). However, the mutation of glycine at position 12 to aspartate (G12D) leads to the projection of a bulkier and negatively charged side group into

\footnotetext{
${ }^{1}$ Department of Genetics and Genomic Sciences, Icahn School of Medicine at Mount Sinai, New York, New York, United States of America. ${ }^{2}$ Icahn Institute for Data Science and Genomic Technology, New York, New York, United States of America. ${ }^{3}$ Department of Chemical and Biological Engineering, College of Engineering, Koç University, Istanbul, Turkey. Correspondence and requests for materials should be addressed to B.E. (email: berman@ku.edu.tr) or Z.H.G. (email: zeynep.gumus@mssm.edu)
} 
A

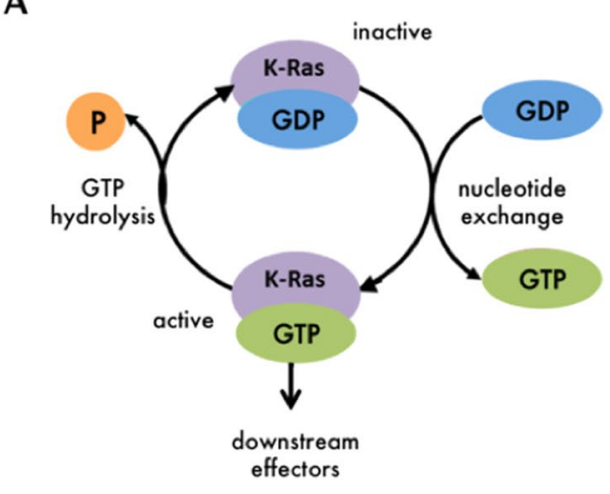
K-Ras-GTP $+\mathrm{H}_{2} \mathrm{O} \longrightarrow \mathrm{K}-$ Ras-GDP $+\mathrm{P}_{\mathrm{i}}$ GTP hydrolysis

D
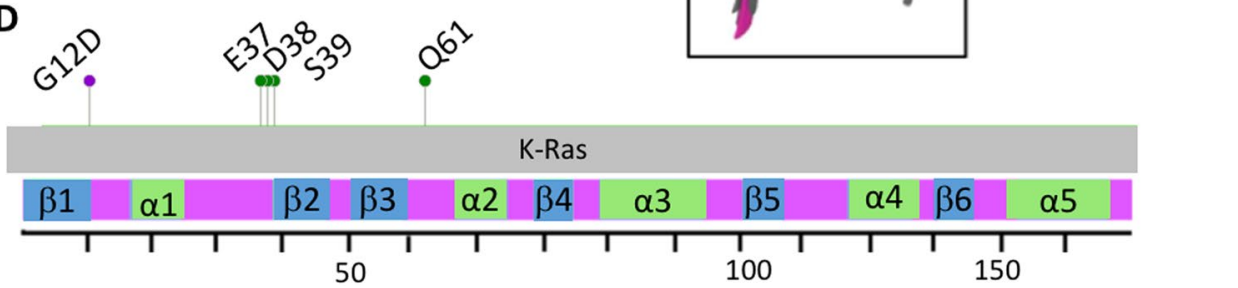

Figure 1. K-Ras switch mechanism and 3D structure. (A) Cartoon representation of K-Ras switch mechanism between GTP-bound (active) to GDP-bound (inactive) forms and the GTP hydrolysis reaction. (B) Cartoon representation of K-Ras protein consisting of secondary structures. Blue: $\beta$-sheets, green: $\alpha$-helices, pink: P-loop, light pink: SI region, purple: SII region. (C) Zoomed-in visualization of the P-loop. Grey: Wild-type, pink: G12D mutant. Residues G12 and D12 are in stick representation. (D) G12D mutation site (red), proteinprotein interaction site residues for its regulator and downstream effector proteins (green) and schematic representation of K-Ras sequence (residues 1-169).

the active site, which causes a steric hindrance in GTP hydrolysis ${ }^{20}$, impairs the GTPase function and locks K-Ras in its active (GTP-bound) state ${ }^{12}$. NMR studies have shown that Ras proteins acquire a range of conformations during their motions in this GTP-bound form ${ }^{21-23}$. Identifying the changes in dynamic conformations of K-Ras upon G12D mutation requires an integrated analysis that targets local conformational and dynamic changes at an atomistic level. Although the effects of G12D mutation on the structure, conformation and flexibility of K-Ras have been studied ${ }^{24-27}$, how it alters the balance of local conformational states, and thereby the local dynamics of K-Ras still remains to be understood. This is an important question, as there is increasing evidence that crystal structure studies alone may miss drug-binding pockets on mutant K-Ras surface, while studies that include dynamics information have recently achieved promising results ${ }^{28-31}$. However, these studies are so far limited to G12C mutant, and local dynamic changes remain unknown. While it is plausible that the development of targeted drugs may remain elusive, understanding the unique dynamic characteristics of the most common oncogenic mutant K-Ras, G12D, can inform research efforts towards this goal.

Here, we present a computational procedure that reveals how the most prevalent oncogenic K-Ras mutation, G12D, triggers structural, conformational and dynamic changes that result in constitutive activation of the protein. Our integrated analysis particularly targets the local changes in the protein structure, conformation and dynamics, since global metrics are mainly affected by loop motions (i.e. rotations and translations) that are not related with its function ${ }^{32}$. We are motivated by recent studies that have utilized protein dynamics data successfully to understand the effects of mutations ${ }^{33-35}$. Most notably in drug discovery, dynamics data on oncogenic proteins ${ }^{25,36-39}$ have helped identify cryptic or allosteric binding sites ${ }^{40-43}$. Encouraged by these results, we have hypothesized that the mutation-specific dynamic behavior of active and inactive K-Ras can best be explored by detailed analyses of their MD simulation data, from which we can investigate the range of their dynamic conformations and their atomistic-scale structural basis. To test our hypothesis, we used an integrated computational analysis that quantifies mutation-based local changes in protein conformations and their dynamic consequences.

In summary, we have performed replicate long time molecular dynamics (MD) simulations (1 microsecond) of both wild-type and G12D mutant K-Ras (K-Ras ${ }^{\mathrm{G} 12 \mathrm{D}}$ ) in GTP-bound (active) and GDP-bound (inactive) forms. Briefly, we first studied the changes in the population of local conformational states caused by the mutation by evaluating the alterations in residue pair distances and local volume. Then, we explored the residue-specific population of conformational states of K-Ras and the population shift upon G12D mutation, and elucidated the structural changes that alter the formation of $\mathrm{H}$-bonds and salt bridges that govern population balance of 
A

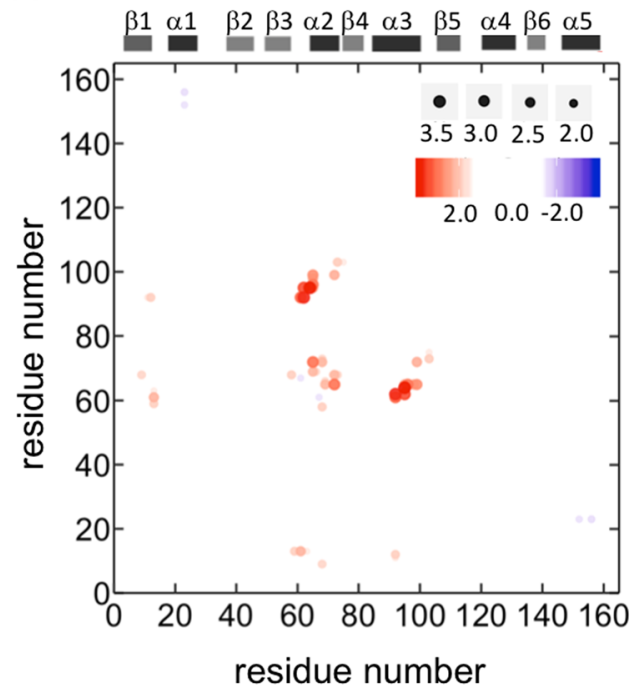

B

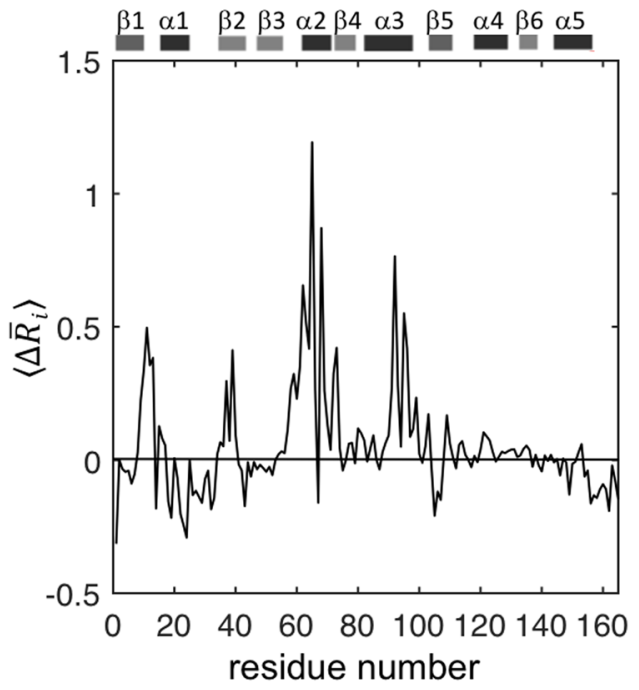

Figure 2. Conformational changes in K-Ras upon G12D mutation. (A) The differences in average pairwise distances $\left(\Delta \bar{R}_{i j}\right)$ where K-Ras ${ }^{\mathrm{WT}}$ is the reference state. Red dots (positive $\Delta \bar{R}_{i j}$ values) show that pairs move further apart and blue dots (negative $\Delta \bar{R} i j$ values) show that pairs move closer in K-Ras ${ }^{\mathrm{G} 12 \mathrm{D}}$. (B) The average of all $\Delta \bar{R}_{i j}$ values for each residue, $\Delta \bar{R} i$. The initial state is $\mathrm{K}-\mathrm{Ras}^{\mathrm{WT}}$ and the final state is K-Ras ${ }^{\mathrm{G} 12 \mathrm{D}}$. The residues which move away from their neighbors have positive values and dominate the mutant protein; residues that move close to their neighbors have negative values.

local conformational states. We then identified changes in local dynamics through a multi-step process where we quantified the fluctuations of all residues; correlated fluctuations for all residue pairs; and identified lost or newly formed correlations upon mutation. Finally, we related the observed structural changes to conformational and dynamic alterations, which enabled us to decode the important local changes that affect K-Ras function due to the G12D mutation. Overall, our study enhances our understanding of K-Ras ${ }^{G 12 D}$ dynamics and can inform studies on the development of direct inhibitors.

\section{Results}

Residue pair distance calculations show that G12D mutation causes local conformational changes in K-Ras. To understand the local conformational changes around each residue upon G12D mutation, we first analyzed the MD simulation data of both active (GTP-bound) and inactive (GDP-bound) forms of wild-type and G12D mutant K-Ras by using conventions from the Gaussian Network Modeling (GNM) approach, a widely employed tool in the analysis of protein dynamics. Specifically, we defined the first coordination shell of a residue as a sphere of radius $\sim 7.2 \AA^{44-47}$, and the second coordination shell as twice the volume of the first, at a radius of $\sim 9.1 \AA^{47}$ (See Methods). We then compared the distances between all residue pairs within their second coordination shells in wild-type vs. mutant K-Ras. For this purpose, we represented the changes in the time-averaged distance between two residues $i$ and $j$ that are within their second coordination shells by $\Delta \bar{R}_{i j}=\bar{R}_{i j, \text { mutated }}-\bar{R}_{i j, W T}$ where $\bar{R}_{i j}$ is the time averaged distance between residues $i$ and $j$. In Fig. $2 \mathrm{~A}$, we show the $\Delta \bar{R}_{i j}$ values for all residue pairs, where K-Ras ${ }^{W T}$ is the reference and $\mathrm{K}-\mathrm{Ras}^{\mathrm{G} 12 \mathrm{D}}$ is the final structure. As seen from the abundance of positive $\Delta \bar{R}_{i j}$ values from Fig. 2A, the dominant distortion of the protein upon mutation is expansion. Specifically, (i) the SII loop (Q61-S65) moves away from $\alpha 3$ (D92-Q99); (ii) the residues A11-G13 (P-loop) move away from Q61H (SII) and (iii) the C-terminal residue of the $\alpha 2$ helix (M72) move away from the N-terminal residues of the same helix $(\mathrm{S} 65, \mathrm{R} 68)$ and $\alpha 3$ (Q99). On the other hand, we observed that some residue pairs became (weakly) closer in K-Ras ${ }^{\mathrm{G} 12 \mathrm{D}}$, including D69 (SII)-V103 ( $\alpha 3$ ), and L23 ( $\left.\alpha 1\right)-\mathrm{V} 152$, and F156 $(\alpha 5)$, demonstrated by their negative $\Delta \bar{R}_{i j}$ values in Fig. $2 \mathrm{~A}$.

In summary, the time-averaged distance analysis disclosed the local conformational changes in K-Ras in response to the G12D mutation. Based on these findings, we next aimed to understand the local effects of this mutation on GTP-bound (active) and GDP-bound (inactive) K-Ras protein dynamics in more detail, specifically focusing on the regions: (i) SII- $\alpha 3$, (ii) P-loop-SII and (ii) $\alpha 1-\alpha 5$.

G12D mutation alters the distribution of conformations of active K-Ras. To better understand the conformational changes upon G12D mutation, we explored the distribution of distances between all residue pairs for both active (GTP-bound) and inactive (GDP-bound) states of K-Ras (Fig. 2A) from their MD simulation data. We observed that the wild-type and mutant proteins exhibited distinct distribution patterns, which suggests that they visited different conformational states as indicated by specific peak points. Specifically, while the residue pair distances in both inactive (GDP-bound) wild-type and mutant K-Ras exhibited broad distributions with multiple peaks (Figs 3 and 4), in active (GTP-bound) K-Ras, they exhibited narrow distributions in wild-type with two distinct peaks. However, active mutant K-Ras shows a predominant conformation ( $>80 \%)$ with a broad distribution. 

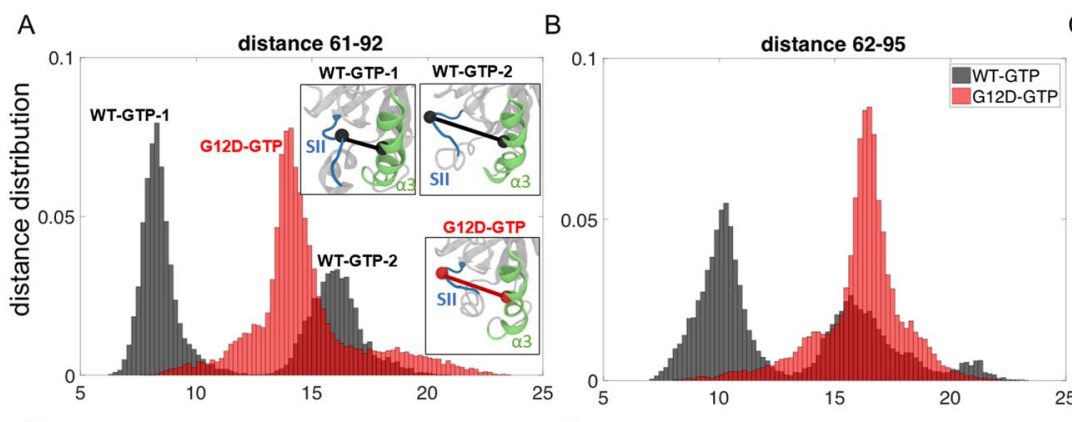

C distance 64-95
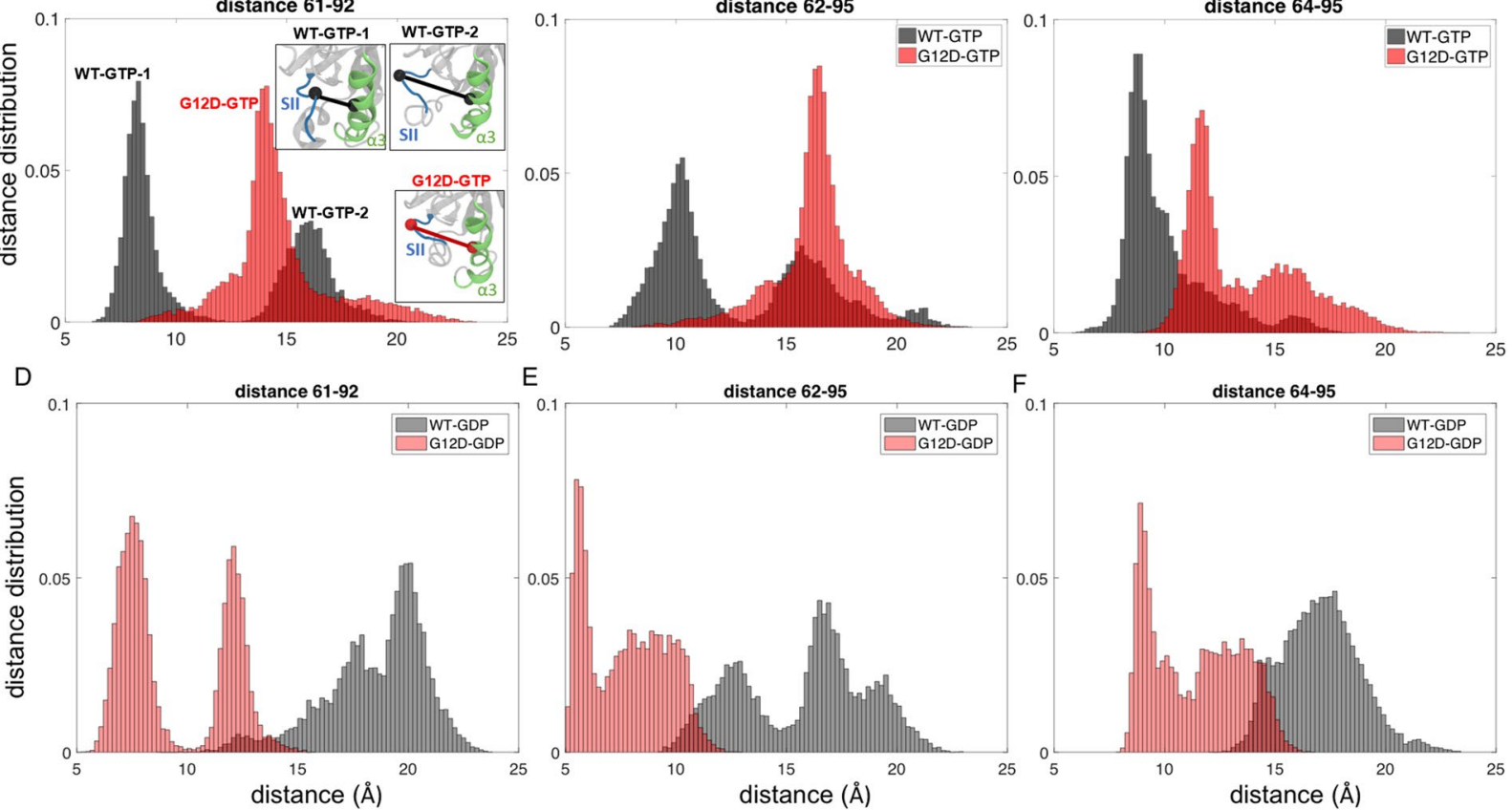

Figure 3. Distribution of distances between residue pairs in SII- $\alpha 3$ region. Distance distributions of C $\alpha$ of residue pairs in K-Ras ${ }^{\text {WT }}$-GTP (black) and K-Ras ${ }^{\text {G12D }}$-GTP (red) (A) Q61-D92, (B) E62-H95, (C) Y64-H95; in K-Ras ${ }^{\text {WT }}$-GDP (grey) and K-Ras ${ }^{\text {G12D }}$-GDP (pink) (D) Q61-D92, (E) E62-H95, (F) Y64-H95.
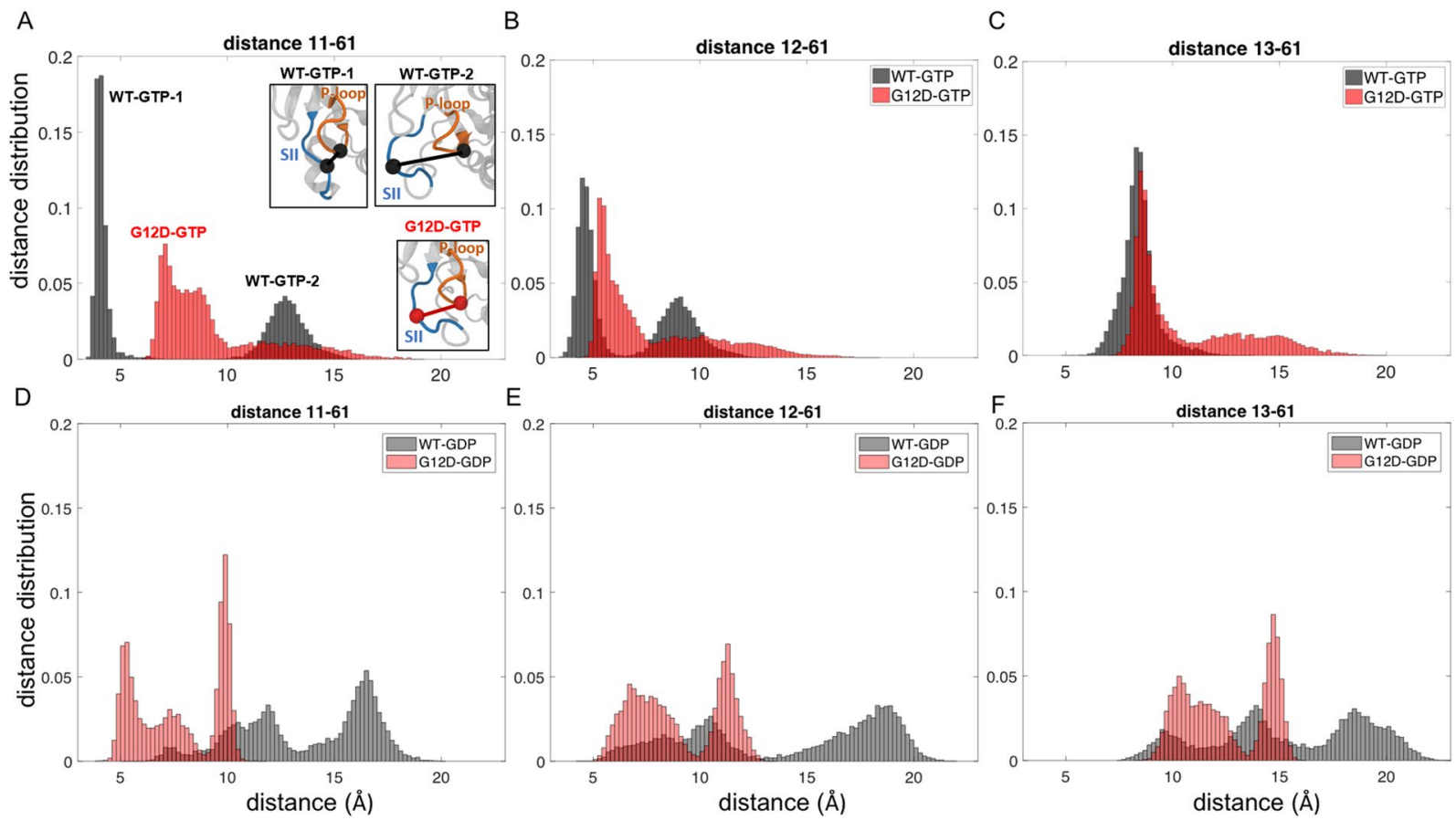

Figure 4. Distribution of distances between residue pairs in the P loop-SII region. Distance distributions between Cos of residue pairs in K-Ras ${ }^{\text {WT }}$-GTP (black) and K-Ras ${ }^{G 12 D}$-GTP (red) (A) A11-Q61, (B) G12D-Q61, (C) G12D-Q61; in K-Ras ${ }^{\text {WT }-G D P ~(g r e y) ~ a n d ~ K-R a s ~}{ }^{\mathrm{G} 12 \mathrm{D}}$-GDP (pink) (D) A11-Q61, (E) G12D-Q61, (F)

G12D-Q61.

G12D mutation alters the balance of local conformational states between SII and $\alpha 3$ regions. Based on our analysis of all residue pairs, the largest conformational change due to G12D mutation in active K-Ras was between SII and $\alpha 3$ regions. We observed that the distances between the residue pairs 61-92, 62-92, 62-95 and 64-95 populate multiple conformations with the peak points at (i) $12.2 \AA, 18.5 \AA$, $20 \AA$ for $61-92$, (ii) 
13.6 $\AA$, $17 \AA$, $19.5 \AA$ for $62-92$ and (iii) $14.7 \AA$, $18.6 \AA$ for $64-95 \AA$ in inactive (GDP-bound) K-Ras ${ }^{\text {WT }}$ (Fig. 3). However, GTP binding causes these pairs to have two distinct conformations, where the peak of the distance distribution of the first conformation is at a shorter distance ( $8.3 \AA$ for $61-92,10 \AA$ for $62-92,8.8 \AA$ for $64-95 \AA)$ and the peak of the second conformation is at a longer distance (16 for 61-92, 15.8 $\AA$ for $62-92,11.6 \AA$ for $64-95 \AA$ ) (Fig. 3A-C). However, G12D mutation leads to a predominant conformation indicated by the peak points at $14 \AA$ for $61-92$ (Fig. 3A) and 16.4 $\AA$ for 62-92 (Fig. 3B), which is similar to the second conformation of active wild-type. Although the pair 64-95 in the mutant protein can visit longer distances, its predominant conformation is indicated by the peak at $11.8 \AA$ (Fig. 3C). Interestingly, the mutation affects the GDP-bound protein differently: while the distances between the residues still populate multiple conformations as in GDP-bound K-Ras ${ }^{\mathrm{G} 12 \mathrm{D}}$, peaks are at shorter distances ((i) $7.5 \AA$ and $12 \AA$ for $61-92$, (ii) $5.5 \AA$ and $9 \AA$ for $62-92$, (iii) $8.8 \AA$ and $12.5 \AA$ for 64-95), and are in fact similar to the first conformation of GTP-bound K-Ras ${ }^{W T}$ (Fig. 3D-F).

G12D mutation driven changes in conformational states between SII and $\alpha 3$ regions are due to the brakeage of $\mathrm{H}$-bonds and the formation of a salt bridge between them. SII region consists of the SII loop (A59-Y64, N-terminus) and the $\alpha 2$ helix (residues S65-T74, C-terminus). In mutant K-Ras-GTP, $\alpha 2$ helix of SII interacts with $\alpha 3$ helix through an H-bond between R68 and D92 in and in the second conformational state of wild-type K-Ras-GTP. Because of the omega shape of the SII loop, the N-terminal residues of SII (Q61, E62 and Y64) assume a distant conformation from $\alpha 3$ helix at the presence of the H-bond between $\alpha 2-\alpha 3$, while the C-terminal residues of SII move toward $\alpha 3$ (D69-V103 pair in Fig S1). In the first conformational state of wild type K-Ras-GTP, this H-bond is not present and thus E62 on the SII loop forms a salt bridge with K88, while K88 forms a salt bridge with D92. The SII loop and $\alpha 3$ helix get closer via these salt bridges. Similarly, in mutant K-Ras-GDP, the formation of an $\mathrm{H}$-bond between the SII loop and $\alpha 3$ helix regions drives these regions to switch between two conformations: a close conformation through the H-bond of E63 (SII)-Q99 ( $\alpha 3$ ) and an open conformation in its absence. However, in wild-type K-Ras-GDP simulations, we did not observe the E63-Q99 $\mathrm{H}$-bond, and the SII loop- $\alpha 3$ residue pairs were further apart.

G12D mutation causes a population shift in the conformations of Q61- P-loop. For inactive (GDP-bound) K-Ras ${ }^{\mathrm{WT}}$, the distance distribution plots between P-loop residues A11-G13 and SII residue Q61 (Fig. 4) show multimodal distributions with the peak points at (i) $7.5 \AA, 12 \AA, 16.5 \AA$ for $11-61$, (ii) $8.5 \AA, 10.5 \AA$, $18 \AA$ for $12-61$ and (iii) $9.5 \AA, 13.8 \AA, 18.5 \AA$ for $13-61 \AA$, indicating three different conformational states (Fig. 4D-F). However, in active (GTP-bound) K-Ras ${ }^{\text {WT }}$ plots (Fig. 4A,B), A11-Q61 and G12-Q61 pairs show only two distinct conformational states indicated by the peak points at (i) $4 \AA$, $13 \AA$ for $11-61$ and (ii) $4.5 \AA, 9 \AA$ for $12-61$, which are similar to those between SII- $\alpha 3$ regions. The first conformation peak is close to an H-bond distance, while the second conformation peak shifts to a longer distance. In contrast, the distances between G13-Q61 show normal distribution with one peak at a longer distance, $8.5 \AA$ (Fig. 4C). This different conformation of G13 relative to Q61 may have resulted from the omega shape of the P-loop, which turns at the C-terminal neighborhood of G12. After the G12D mutation, the two distinct conformational states of A11-Q61 and D12-Q61 pairs disappear, replaced with a predominant conformation (Fig. 4A, B). While the pairs can obtain distant conformations, they only visit such states for $\sim 22 \%$ of time. In active (GTP-bound) mutant K-Ras, these two pairs, in addition to G13-Q61, exhibit broad distance distributions (Fig. 4A-C). Upon inactivation of mutant K-Ras, these three pairs can obtain multiple conformations similar to the inactive wild-type K-Ras as evident from the peak points at (i) $5.3 \AA$, $7.4 \AA$, $9.8 \AA$ for $11-61$, (ii) $7 \AA$, $11.2 \AA$ for $12-61$ and (iii) $10.3 \AA$, $11.3 \AA$, $14.5 \AA$ for $13-61$ in Fig. $4 \mathrm{D}-\mathrm{F}$.

G12D mutation leads to the formation of a permanent salt bridge between the P-loop and SII regions in active protein. In wild-type K-Ras-GTP simulations, we observed that a salt bridge between K16 $(\alpha 1)$ and E63 (SII) leads to two distinct conformations of the P-loop-SII pairs. Residue K16 resides at the $\mathrm{C}$-terminal end of the P-loop, while the residues A11 and G12 reside at its N-terminus. When K16 forms the salt bridge with E63 (SII), A11-G12 get distant from Q61 (SII) corresponding to the second confirmation in Fig. 4, and when this salt bridge dissappers, A11-G12 get closer to Q61 as in the first conformation. However, in mutant K-Ras-GTP simulations, this salt bridge does not disappear. Furthermore, K16 forms an H-bond with G10 on the P-loop (note that the mutated residue at position 12 is also on this P-loop). Because of this H-bond, A11-D12 and Q61 pairs are located at a distance between the first and the second conformations of A11-G12 and Q61 pairs in wild-type (Fig. 4A-C).

G12D mutation causes $\alpha 5$ helix to move towards $\alpha 1$ helix in active K-Ras. In addition to the residue pairs that assume distant conformations after mutation, we also investigated the distance distributions of the $\alpha 1$ and $\alpha 5$ regions, and observed that they got closer in active K-Ras ${ }^{\mathrm{G} 12 \mathrm{D}}$ (Fig. 5). Specifically, we observed that in active K-Ras ${ }^{\text {WT }}$, the residue pair distance distribution curves for L23 ( $\left.\alpha 1\right)-\mathrm{V} 152(\alpha 5)$ and L23 ( $\left.\alpha 1\right)$-F156

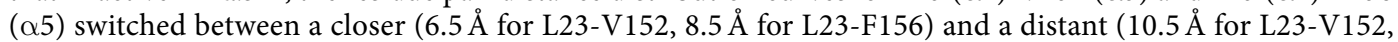
$14 \AA$ for L23-F156) conformational state. Furthermore, the inactive wild-type and inactive mutant proteins had similar patterns in their distribution curves for these residue pairs. However, in the active K-Ras ${ }^{G 12 D}$, the distance distribution curves of these pairs had single peaks (6.5 ̊ for L23-V152, 8.5 ̊ for L23-F156), similar to their closer conformational states in active K-Ras ${ }^{\mathrm{WT}}$.

G12D mutation leads to changes in conformations of residues relative to their neighborhood. According to GNM, a residue typically fluctuates within its first or second coordination shells $\mathrm{s}^{44,45}$. Within these volumes, there are several other residues, which are either near-neighbors along the chain or are spatially distant. As has been shown by the GNM model ${ }^{45}$, a residue with a smaller number of neighbors will show larger fluctuations than a residue with a larger number of neighbors. Therefore, the neighborhood of a given residue significantly affects its fluctuations and dynamics. To understand which parts of K-Ras move away from its neighbors 

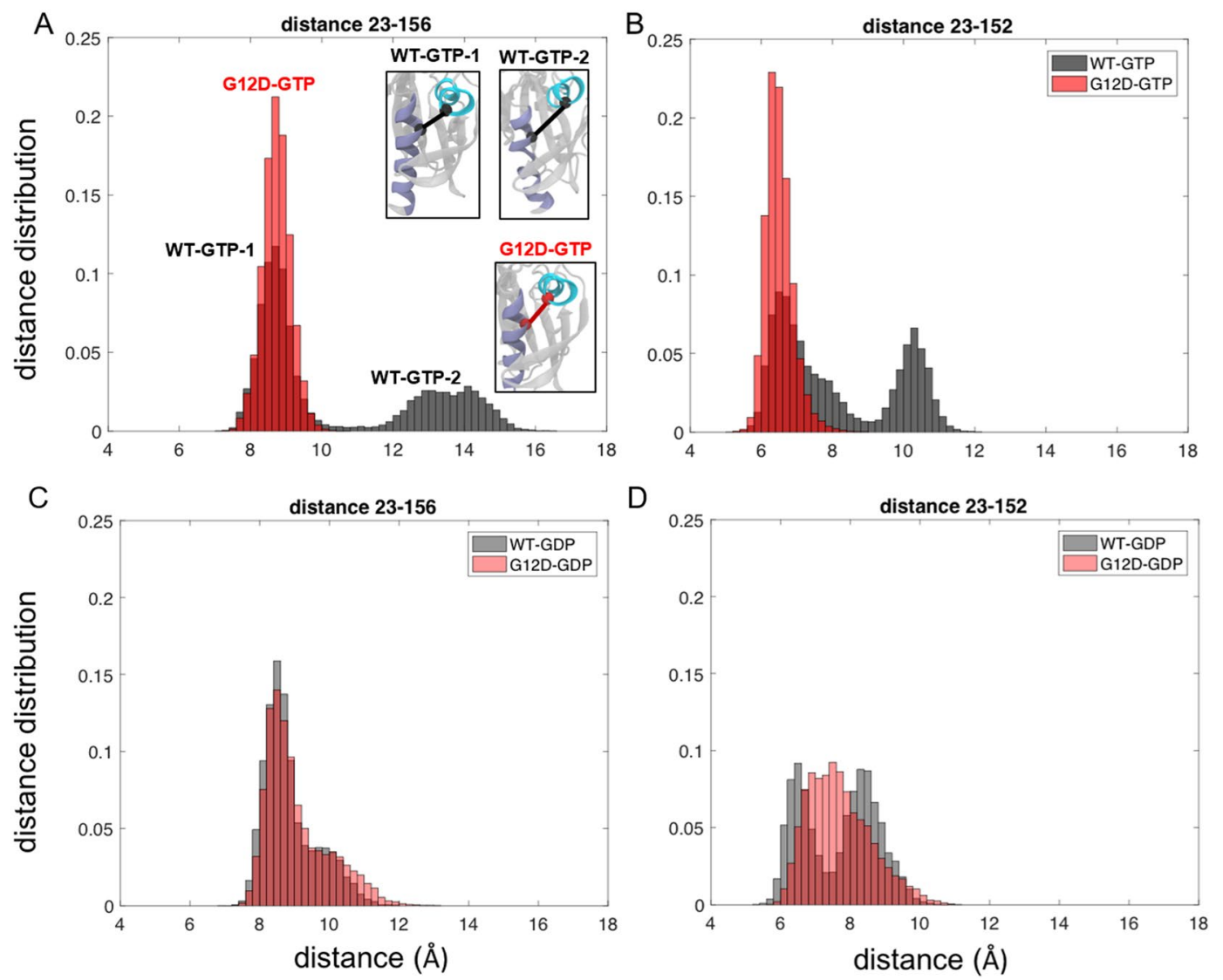

Figure 5. Distance distributions of residue pairs which get closer after G12D mutation in K-Ras-GTP in K-Ras ${ }^{\text {WT }}$ (black) and K-Ras ${ }^{\mathrm{G} 12 \mathrm{D}}$ (red). Distance distribution of Cos of residue pairs in K-Ras ${ }^{\mathrm{WT}}$-GTP (black) and K-Ras ${ }^{\mathrm{G} 12 \mathrm{D}}$-GTP (red) (A) L23-V152, (B) L23-F156; in K-Ras ${ }^{\mathrm{WT}}$-GDP (grey) and K-Ras ${ }^{\mathrm{G} 12 \mathrm{D}}$-GDP (pink) (C) L23-V152, (D) L23-F156.

and which parts move closer upon mutation globally, we calculated the average of all $\Delta \bar{R}_{i j}$ values (the time averaged distance between two residues $i$ and $j$ ) for each residue $i, \Delta \bar{R} i$. Overall, we observed that after the G12D mutation, most protein parts, especially the P-loop, SI, SII and $\alpha 3$, move away from their neigbors, suggesting larger fluctuations (Fig. 2B).

We then aimed to understand the relation between the changes in conformations of a residue pair and the changes in individual conformations of each residue in that pair. In the distance distribution calculations (Figs 3-5, S1), we present the results of the residue pairs that underwent the largest changes in distance due to G12D mutation. For each residue in the identified pairs, we estimated the extent of its deviation from its neighbors by comparing the individual $\sum_{j} \Delta \bar{R} i j$ values of the residues in the identified pairs (Supplementary Table S1). We discovered that distant residue pairs, which move further away from each other in K-Ras ${ }^{\mathrm{G} 12 \mathrm{D}}$ also move away from their proximal neighbors. On the other hand, residue pairs that move closer to each other in K-Ras ${ }^{\mathrm{G} 12 \mathrm{D}}$ assume closer conformations relative to their proximal neighbors.

G12D mutation leads to increased fluctuations of loop residues in SII region. Next, to understand how the flexibility of K-Ras changes upon G12D mutation, we calculated the root-mean-square fluctuations (RMSF) of each residue in both wild-type and mutant protein, where RMSF is a measure of the average atomic fluctuations of a residue.

First, we investigated the effects of the mutation on active wild-type and mutant protein flexibilities, and observed that in the mutant protein, the fluctuations of the loop residues of SII are increased (Fig. 6A). Our residue pair distance calculations in active mutant K-Ras also showed that the residues in SII loop move away from some of the residues in $\alpha 3$ helix, and distances between those residue pairs display broad distributions (Fig. 3). Considering the increased fluctuations of SII, these broad distance distributions with larger peak values between SII and other parts of the protein may be arising from the increased flexibility of SII due to G12D mutation.

We then calculated the effects of mutation on inactive K-Ras flexibility by comparing residue fluctuations of wild-type and mutant protein. Figure 6B shows that the effect of G12D mutation on inactive (GDP-bound) protein is opposite to its effect on the active (GTP-bound) form, where the SII loop residue fluctuations are decreased after G12D mutation in K-Ras-GDP. Furthermore, SI fluctuations are also decreased in mutant K-Ras-GDP. 

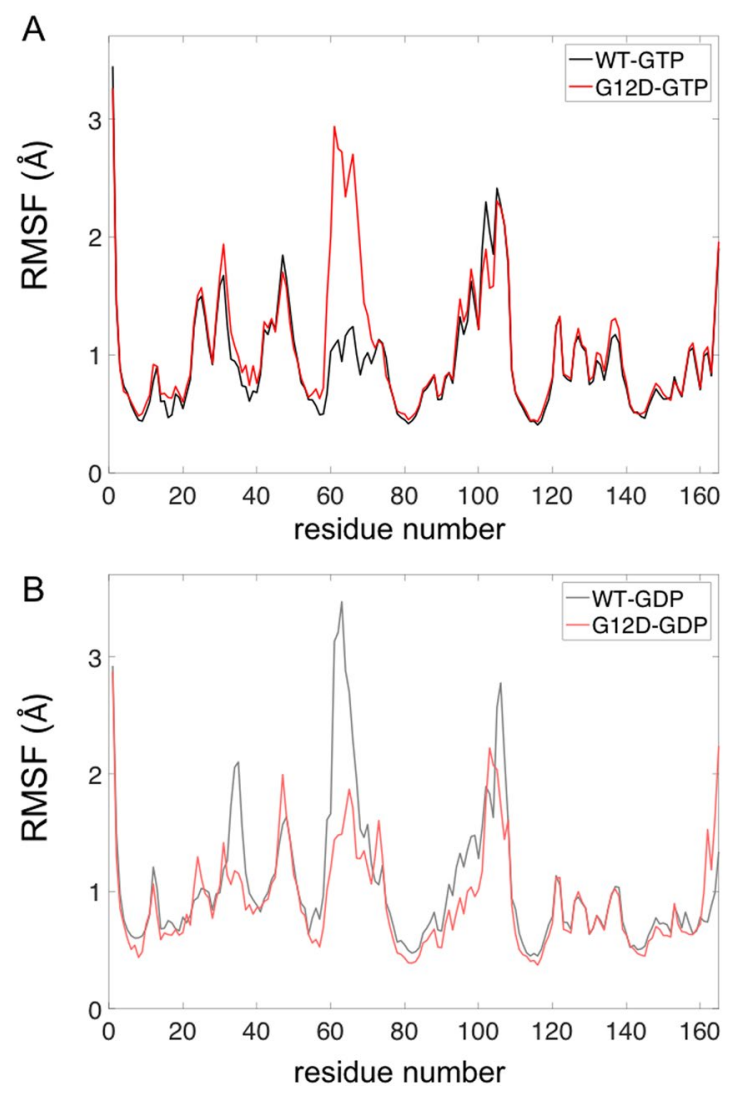

Figure 6. Dynamic changes in K-Ras upon G12D mutation. (A) RMSF values of K-Ras ${ }^{\text {WT }}$-GTP (black) and $\mathrm{K}-\mathrm{Ras}^{\mathrm{G} 12 \mathrm{D}}$-GTP (red) residues. (B) RMSF values of K-Ras ${ }^{\mathrm{WT}}$-GDP (grey) and K-Ras ${ }^{\mathrm{G} 12 \mathrm{D}}$-GDP (pink) residues.

G12D mutation leads to increased SIl loop fluctuations in the active protein, making it unable to form salt bridges. From the analysis of the MD simulation datasets, we observed that E63 (SII) forms a salt bridge with R68 (SII) in active form in wild-type K-Ras, but not in active mutant. The increased fluctuations of the SII loop in the absence of E63-R68 bond are shown in Fig. 6A. However, in inactive mutant, this salt bridge is intact and the fluctuations of the SII loop are decreased, as shown in Fig. 6B.

G12D mutation markedly increases the negatively correlated motions of SII residues in active K-Ras. Regulation of protein dynamics is strictly coordinated by the correlations of residue fluctuations. Figure 7 presents pairwise correlations of residue fluctuations $\left(C_{i j}\right.$ ), for K-Ras ${ }^{\text {WT }}$-GTP (upper-left),

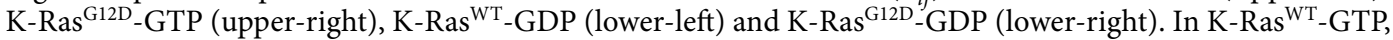
$\beta 2-\beta 3$ regions move in negative correlation with $\alpha 1-S I$ region and $\beta 6$; but this correlation is disturbed by the G12D mutation. However, the most striking effect of the mutation on the active protein is the occurrence of new correlations between the motions of the SII loop and other protein regions. As seen in Fig. 7B, upon G12D mutation, motions of residues in SII become negatively correlated with those of residues in P-loop, $\beta 3, \beta 4$ and $\alpha 3$-Loop7. For the inactive GDP-bound form, G12D mutation does not have a notable effect on motion correlations.

G12D mutation does not affect the coupled motions of the central $\beta$-sheet. As seen in Fig. 1, $\mathrm{K}$-Ras structure consists of a central $\beta$-sheet that is composed of six $\beta$-strands, which are surrounded by five $\alpha$-helices. Our correlation calculations show that the $\beta$-strands fluctuate in correlation in both the active and inactive wild type forms (Fig. 7A, C). Specifically, motions of $\beta 1$ are coupled to motions of the other strands; $\beta 2$ and $\beta 3$ motions are coupled to each other and motions of $\beta 5$ are coupled to motions of $\beta 4$ and $\beta 6$. These coupled motions within the central $\beta$-sheet are not altered by the G12D mutation.

G12D mutation causes negatively correlated fluctuations in regions (Fig. 7B) that move away from each other in inactive protein. We observed that after G12D mutation, negative correlations occur between the regions -SII-P-loop and SII- $\alpha 3$ - which move away from each other (Fig. 2A) and from their neighbors (Fig. 2B). The combination of distance (Fig. 2) and correlation (Fig. 7B) calculations reveals the relation between conformational and dynamic changes in the protein as a result of the G12D mutation. 
A

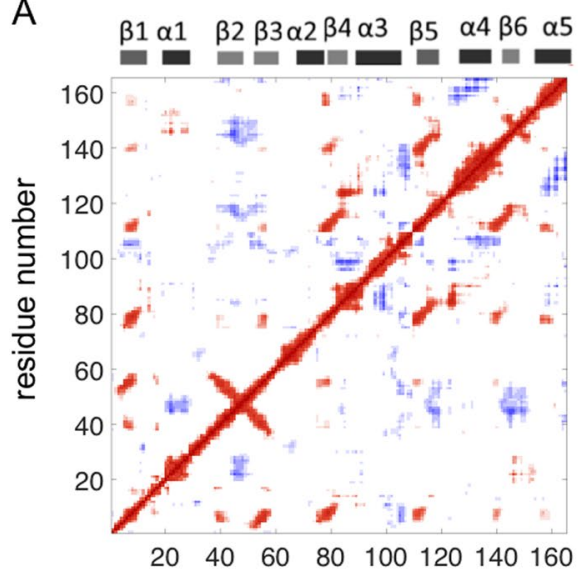

C

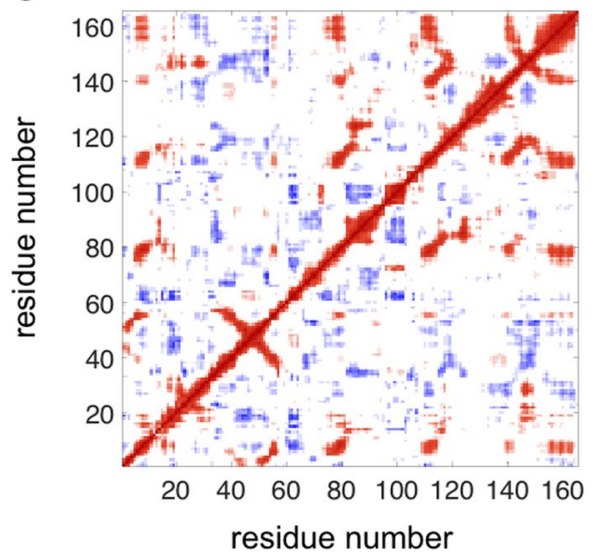

B

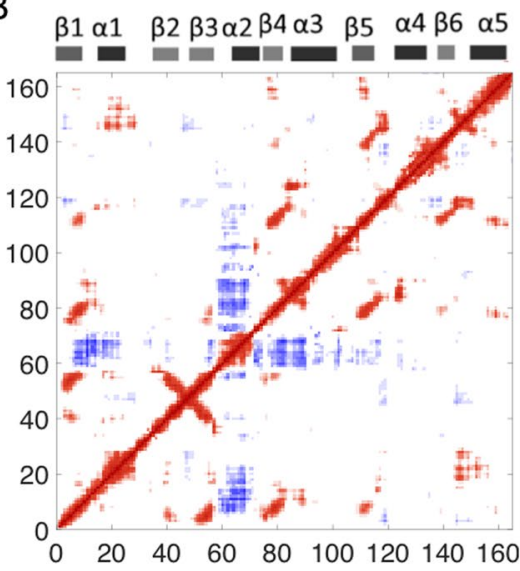

$\mathrm{D}$

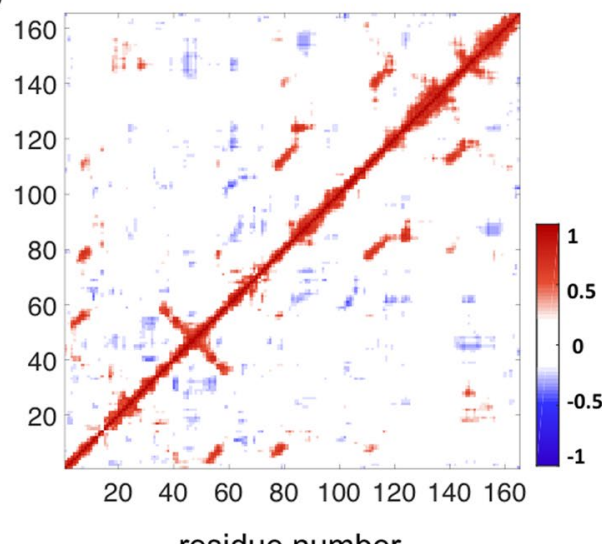

residue number

Figure 7. The maps of correlation coefficients from the motions of correlated residues in (A) K-Ras ${ }^{\mathrm{WT}}$-GTP, (B) K-Ras ${ }^{\mathrm{G} 12 \mathrm{D}}$-GTP, (C) K-Ras ${ }^{\mathrm{WT}}$-GDP and (D) K-Ras ${ }^{\mathrm{G} 12 \mathrm{D}}$-GDP. Red dots show positive correlations and blue dots show negative correlations.

\section{Discussion}

K-Ras is an important GTPase in cellular signaling that is only active in its GTP-bound state ${ }^{8,9}$. Structurally, in wild-type active K-Ras, the P-loop, SI and SII switch regions are bound to the phosphate groups of GTP and are responsible for its GTPase function. However, when there is a G12D mutation in the P-loop, GTP hydrolysis is impaired and K-Ras freezes in its active state ${ }^{9}$, causing uncontrollable cellular growth and evasion of apoptotic signals ${ }^{27,48,49}$. Despite extensive literature on the effects of G12D mutation on K-Ras, its effects on the regulation of local dynamics of the active and inactive protein, and how these relate to its effects on structure and local conformations of the protein remain unknown. At the same time, since protein function is intrinsically related to its dynamics, this information can support studies on effective targeting of mutant K-Ras. To understand the local changes in the dynamic behavior of K-Ras caused by the G12D mutation, we first identified the structural and conformational changes in its residues and then related them to changes its dynamic characteristics by MD simulation data analysis of both wild-type and mutant K-Ras in GTP- and GDP-bound forms.

Using residue pair distance analysis, we have discovered that K-Ras accesses a range of conformational states during its simulations, and that the G12D mutation alters the distribution of its conformational states in a bound-nucleotide specific fashion. Overall, the residue pair conformational states, which we present in Figs 3-5, populate multiple distances in inactive (GDP-bound) wild-type and mutant K-Ras. However, they adopt two different conformational states in active (GTP-bound) wild-type protein and only a single predominant conformational state in active mutant protein. Specifically, the residue pairs between SII- $\alpha 3$ have two different conformational states, which we have labeled as first and second conformational states in Results. While the wild-type protein populates both conformational states, the second conformation is predominant in the G12D mutant. In the first conformation, the N-terminus of SII is positioned toward N-terminus of $\alpha 3$ helix (Fig. 3), and the central residues of SII move away from $\mathrm{C}$ terminal end of $\alpha 3$ (Fig. S1). In this conformation, we observed an $\mathrm{H}$-bond network within SII residues: (i) R68 forms bonds with E63, S65, Y71, M72, (ii) M67 is bound to Q70 and (iii) Q61 is bound to E63. However, in the second conformation, the central residues of SII move toward C terminal end of $\alpha 3$, this H-bond network disappears and SII forms H-bonds with $\alpha 3$.

Our observation of two conformational states for active wild-type K-Ras is consistent with previous experimental studies ${ }^{50-54}$, which have shown that the protein has two states, a catalytically incompetent $(\mathrm{T})$ and a catalytically active (R) state, and that SII can assume a variety of positions due to the conformations of $\alpha 3$ helix and 
loop L7 that associate with these states (please note that as shown in Fig. 1, L7 is right adjacent to $\alpha 3$, where $\alpha 3$ ends at residue 103 and the loop begins at residue 104). Briefly, these studies have shown that in "T" state, $\alpha 3-\mathrm{L} 7$ moves toward SII and disrupts the H-bond network centered at R68 in SII ${ }^{55}$. Downstream signaling remains 'on', as GTP hydrolysis with disordered SII region is slow. On the other hand, in " $\mathrm{R}$ " state, H-bond network in SII is intact, allowing for catalytic activity and turning K-Ras signaling "off”. Our results provide an atomistic level structural explanation to these observations by revealing that G12D mutation shifts the two SII- $\alpha 3$ conformations in favor of a single predominant conformation (the second conformation), disrupting the H-bond network between SII residues (Fig. 3). In summary, our results suggest that the deviation of central SII residues toward $\alpha 3$ helix, and the disruption of the H-bond network within SII may impair GTP hydrolysis, leading to the constitutive activation of K-Ras ${ }^{\mathrm{G} 12 \mathrm{D}}-\mathrm{GTP}$ signaling.

The importance of communication between SII and $\alpha 3$ has also been shown in other studies, which further cumulatively suggest that the conformational landscapes of Ras proteins may be mutation- or isoform- specific. The first study that identified unique interactions between SII and $\alpha 3$ (M67-Q99/I100, F78/C80-I100) was published by Gorfe et al. ${ }^{56}$. Then, several studies identified multiple SII- $\alpha 3$ conformations in GTP-bound K-Ras. Specifically, Buhrman et al. discovered a shift in coupled SII and a3-L7 conformations in all GTP-bound Ras isoforms and defined this mechanism as an "allosteric switch". Furthermore, Prakash et al., showed a displacement of $\alpha 3$ (Y96) away from SII(G60) during GTP-to-GDP transition by sampling K-Ras Q61 mutant conform$\mathrm{ers}^{57}$. Consistently, we also observed multiple conformations of the SII- $\alpha 3$ pair in the wild-type protein (Fig. 3). However, the number of conformations decreased upon G12D mutation as shown with the distribution of SII- $\alpha 3$ distances in Fig. 3. Recent studies support such mutational effects on SII- $\alpha 3$ conformations. Sayyed-Ahmad et al. observed that Y71 interacts with Y96 in most mutants and that R68 and Y96 are engaged in a stable interaction in K-Ras, which is similar to our observation on mutant K-Ras (Fig. 3), in which $\alpha 2$ helix of SII interacts with $\alpha 3$ helix through an $\mathrm{H}$-bond between $\mathrm{R} 68$ and $\mathrm{D} 92^{58}$. Pantsar et al. analyzed the long-time scale dynamics of multiple K-Ras G12 mutants and identified a hub network that includes SII (M72, F78, L79) and $\alpha 3$ (F90, I100) residues ${ }^{59}$, where SII (M72) of GTP-bound G12D mutant displayed interactions almost identical to those of the wild-type. Consistent with their finding, we also observed that SII- $\alpha 3$ pair in K-Ras ${ }^{\mathrm{G} 12 \mathrm{D}}$-GTP populates at a conformation that is similar to its second conformation in the wild-type protein (Fig. 3).

In our residue pair distance analyses, we also observed that in wild-type K-Ras-GTP, the distribution curves of the distances between the P-loop and Q61 are Gaussian-shaped, narrow dispersion curves. However, in G12D mutant K-Ras-GTP, they significantly deviate from the Gaussian. Since Q61 is a known critical catalytic residue for both intrinsic and GAP-mediated GTP hydrolysis, it is possible that this highly variable nature of the P-loop-Q61 distance, suggesting high flexibility, affects GTP hydrolysis in K-Ras G12D. Furthermore, using the average of residue pair distances for each residue, $\Delta \bar{R} i$, we observed that the P-loop, SI, SII and $\alpha 3$ regions move away from their neighbors upon G12D mutation (Fig. 2B).

In the MD simulations, we observed that K-Ras G12D mutation further alters the H-bond network and formation of salt bridges. Because of the formation and breakage of the bonds between different parts of the protein, the P-loop, SII and $\alpha 3$ regions of mutant K-Ras assume conformations different than the wild-type. Furthermore, by correlating the conformational and dynamic changes in residues at these regions, we discovered that the G12D mutation leads to the coupling of the motions of SII region with those of the residues at these sites. The effects of the G12D mutation in increasing these correlations are clearly observed in pairwise correlation maps (Fig. 7B), which show marked differences between K-Ras ${ }^{\mathrm{G} 12 \mathrm{D}}$ and K-Ras ${ }^{\mathrm{WT}}$, and are also consistent with the increased amplitude of SII fluctuations, as shown in Fig. 6. Our results are consistent with a previous computational study that showed that SII displays increased fluctuations and negative correlations with other parts of the protein ${ }^{27}$. Consequently, we show that the G12D mutation leads to characteristic population of relative SII conformations and also changes in SII flexibility and dynamics. Our study goes beyond this by identifying the atomistic basis of the changes in dynamic behavior of K-Ras in terms of bond formations.

Overall, these results provide a new understanding of the local changes in dynamic behavior of K-Ras ${ }^{\mathrm{G} 12 \mathrm{D}}$ and an atomistic basis for this behavior. Such an understanding can support studies that target the protein with small molecules, which can be an effective strategy for the ever elusive allosteric inhibition of oncogenic K-Ras ${ }^{\mathrm{G} 12 \mathrm{D}}$.

\section{Methods}

MD Simulations. We performed all-atom MD simulations for both GTP-and GDP-bound forms of K-Ras ${ }^{\text {WT }}$ and K-Ras ${ }^{\mathrm{G} 12 \mathrm{D}}$. We obtained the K-Ras-GTPWT and K-Ras-GDPWT structures from the final frame of the simulations of active and inactive states proteins by Vatansever et al., respectively ${ }^{60}$. For constructing K-Ras ${ }^{\mathrm{G} 12 \mathrm{D}}$ structure, we mutated glycine to aspartate at position 12 in K-Ras-GTPWT structure using Discovery Studio 4.5 software, (DS) ${ }^{61}$. To optimize the K-Ras ${ }^{\mathrm{G} 12 \mathrm{D}}$-nucleotide complex, we used Clean Geometry tool of DS. For MD simulations, we used NAMD $2.10^{62}$ with AMBER ff99SB ${ }^{63}$ and general amber force fields (GAFF) ${ }^{64}$. Briefly, we performed energy minimization of the initial model after we introduced the G12D mutation in K-Ras, and then ran MD simulations of each complex following the protocols from Vatansever et al. ${ }^{60}$, the details of which we provide in Supplementary Methods. During the simulations, we applied minimization for 10,000 steps and equilibration for 500,000 steps, after which we performed 1 microsecond MD simulations, and saved atomic coordinates $\hat{R}$ of all atoms every $10 \mathrm{ps}$. We repeated the same simulation steps for two independent simulations of each complex. We used the last $900 \mathrm{~ns}$ of the simulation trajectories in all computations described in this study. To eliminate all rotational and translational motions, we aligned the trajectories to the first frame using VMD software 1.9. $2^{65}$. We visualized the trajectories with VMD. To identify salt bridges formed in the protein during the MD simulations, we used Salt Bridges Plugin, Version 1.1, of VMD.

Principal component analysis and projection. Consider a protein with $\mathrm{N}$ residues (for K-Ras protein $\mathrm{N}=165$ ), where the position vector for the $i^{\text {th }}$ atom, $r_{i}=\left[x_{i} y_{i} z_{i}\right]$, is known from simulations performed for $M$ 
time steps. First, we mean-center the coordinate vector by subtracting the temporal averages from each of its coordinates, i.e.,

$$
\bar{r}_{i} \equiv\left[\bar{x}_{i}\left(t_{m}\right) \bar{y}_{i}\left(t_{m}\right) \bar{z}_{i}\left(t_{m}\right)\right]=r_{i}\left(t_{m}\right)-\frac{1}{M} \sum_{k=1}^{M} \bar{r}_{i}\left(t_{k}\right)
$$

Then, we calculate the magnitude (|| ||) of the coordinate vector:

$$
\left\|\bar{r}_{i}\left(t_{m}\right)\right\|=\sqrt{\bar{x}_{i}^{2}\left(t_{m}\right) \bar{y}_{i}^{2}\left(t_{m}\right) \bar{z}_{i}^{2}\left(t_{m}\right)}
$$

and represent the MD simulation data performed for $M$ time steps in an $N x M$ matrix:

$$
\Delta \bar{R}=\left[\begin{array}{cc}
\left\|\bar{r}_{1}\left(t_{1}\right)\right\| & \left\|\bar{r}_{1}\left(t_{2}\right)\right\| \ldots\left\|\bar{r}_{1}\left(t_{M}\right)\right\| \\
\left\|\bar{r}_{2}\left(t_{1}\right)\right\| & \left\|\bar{r}_{2}\left(t_{2}\right)\right\| \ldots\left\|\bar{r}_{2}\left(t_{M}\right)\right\| \\
\vdots & \\
\left\|\bar{r}_{N}\left(t_{1}\right)\right\| & \left\|\bar{r}_{N}\left(t_{2}\right)\right\| \ldots\left\|\bar{r}_{N}\left(t_{M}\right)\right\|
\end{array} \mid\right.
$$

Then, we calculate the covariance matrix $(C)$, which is an $N \mathrm{x} N$ symmetry matrix, by taking the dot product of $\Delta \bar{R}$ and $\Delta \bar{R}^{T}$ as below:

$$
\begin{aligned}
C & =\left\langle\Delta \bar{R} \cdot \Delta \bar{R}^{T}\right\rangle \\
& =\frac{1}{M} \times\left[\begin{array}{c}
\left\|\bar{r}_{1}\left(t_{1}\right)\right\|\left\|\bar{r}_{1}\left(t_{2}\right)\right\| \cdots\left\|\bar{r}_{1}\left(t_{M}\right)\right\| \\
\left\|\bar{r}_{2}\left(t_{1}\right)\right\|\left\|\bar{r}_{2}\left(t_{2}\right)\right\| \cdots\left\|\bar{r}_{2}\left(t_{M}\right)\right\| \\
\vdots \\
\left.\left\|\bar{r}_{N}\left(t_{1}\right)\right\|\left\|\bar{r}_{N}\left(t_{2}\right)\right\| \cdots\left\|\bar{r}_{N}\left(t_{M}\right)\right\|\right|_{N x M}
\end{array}\right] \cdot\left[\begin{array}{c}
\left\|\bar{r}_{1}\left(t_{1}\right)\right\|\left\|\bar{r}_{2}\left(t_{1}\right)\right\| \cdots\left\|\bar{r}_{N}\left(t_{1}\right)\right\| \\
\left\|\bar{r}_{1}\left(t_{2}\right)\right\|\left\|\bar{r}_{2}\left(t_{2}\right)\right\| \cdots\left\|\bar{r}_{N}\left(t_{2}\right)\right\| \\
\vdots \\
\left.\left\|\bar{r}_{1}\left(t_{M}\right)\right\|\left\|\bar{r}_{2}\left(t_{M}\right)\right\| \cdots\left\|\bar{r}_{N}\left(t_{M}\right)\right\|\right|_{M x N}
\end{array}\right.
\end{aligned}
$$

Therefore, each element of the covariance matrix $C_{i j}$ is computed from:

$$
C_{i j}=\frac{1}{M} \sum_{m=1}^{M} \Delta \bar{R}_{i}\left(t_{m}\right) \Delta \bar{R}_{j}\left(t_{m}\right) \quad i, j=1,2, \ldots N
$$

where $\Delta \bar{R}_{i}\left(t_{m}\right)$ denotes the $i^{\text {th }}$ component of $\Delta \bar{R}\left(t_{m}\right)$. Since $C$ is a symmetry matrix, it can be diagonalized with a symmetric eigenvalue decomposition as $C=V \Lambda V^{\mathrm{T}}$, where $\mathrm{V}=\left(\mathrm{V}_{1}, \mathrm{~V}_{2}, \ldots, \mathrm{V}_{\mathrm{N}}\right)$ is a set of real-valued eigenvectors, $\Lambda$ is a real diagonal matrix, and the eigenvector $V_{i}$ corresponds to the eigenvalue $\Lambda_{i i}$ and the $i^{\text {th }}$ column vector of $\mathrm{V}$ is the $i^{\text {th }}$ PCA mode. The projection of simulation data $\Delta \bar{R}\left(t_{m}\right)$ onto the $i^{\text {th }}$ PCA mode is computed from:

$$
P C A_{i}\left(t_{m}\right)=V_{\mathrm{i}} \cdot \Delta \bar{R}\left(t_{m}\right)^{T}=\left[\begin{array}{c}
V_{\mathrm{i} 1} \\
V_{\mathrm{i} 2} \\
\vdots \\
V_{\mathrm{iN}}
\end{array}\right] \cdot\left[\Delta \bar{R}_{1}\left(t_{m}\right) \Delta \bar{R}_{2}\left(t_{m}\right) \cdots \Delta \bar{R}_{N}\left(t_{m}\right)\right]=\sum_{n=1}^{N} V_{\mathrm{i} n} \Delta \bar{R}_{n}\left(t_{m}\right)
$$

Iterating this $P C A_{i}\left(t_{m}\right)$ computation over $m$ for all $M$ time intervals, we obtain the time series $P C A_{i}=\left[P C A_{i}\left(t_{1}\right) P C A_{i}\left(t_{2}\right) \ldots P C A_{i}\left(t_{M}\right)\right]$ for $i^{\text {th }}$ mode. Specifically, we calculated the first $\left(P C A_{1}\right)$ and the second $\left(\mathrm{PCA}_{2}\right)$ modes for projection of MD trajectories onto principal components (Supplementary Fig. S3). Based on the PCA projection plots, we separated the $M$ time points of the trajectories into PCA states, determined the most densely populated states and calculated the probabilities of these states. In calculations of the residue pair distances and correlations, we used the trajectory points in the most densely populated PCA state and weighted the calculations by the probability of its state.

Residue pair distance calculations. To quantify the effect of the G12D mutation on the distances between K-Ras residue pairs, we developed a new computational algorithm detailed in Supplementary Fig. S2. Briefly, we first assumed K-Ras ${ }^{W T}$ as the initial state and $\mathrm{K}-\mathrm{Ras}^{\mathrm{G} 12 \mathrm{D}}$ as the final state. Then, we calculated the distances between $\mathrm{C} \alpha$ atoms of two residues $(i, j)$ as we previously described ${ }^{60}$ and listed in Supplementary Methods. Then we borrowed the 'first coordination shell' definition from the Gaussian network model (GNM), which is widely used in the analysis of protein dynamics. Studies that use GNM typically assume the 'first coordination shell' as the maximum $C \alpha-C \alpha$ distance for the separation between two contacting residues at $\sim 7.2 \AA^{44-47}$. We followed this protocol and determined the first coordination shell around a selected residue by choosing its $\mathrm{C} \alpha$ as the center of a volume $\mathrm{V}$ with a radius of $\mathrm{r} 1 \sim 7.2 \AA^{45}$. However, because the contribution of non-bonded pairs to higher-order coordination shells may also be significant ${ }^{47,66}$, we also studied residue pairs that are within their 'second coordination shell' in K-Ras ${ }^{\mathrm{WT}}$ structure, which we defined at twice the volume of the first, with a radius of $\sim 9.1 \AA^{47}$.

For every residue pair $(i, j)$ where $j$ is in the second coordination shell of $i$, we first calculated its time-averaged distance in K-Ras ${ }^{\mathrm{WT}}\left(\bar{R}_{i j \mathrm{WT}}\right)$ and in K-Ras ${ }^{\mathrm{G} 12 \mathrm{D}}\left(\bar{R}_{i j \mathrm{G} 12 \mathrm{D}}\right)$. We then calculated the difference $(\Delta \bar{R} i j)$ between $\bar{R}_{i j}$ WT and $\bar{R}_{i j \mathrm{G} 12 \mathrm{D}}$, where $\Delta \bar{R} i j=\bar{R}_{i j \mathrm{G} 12 \mathrm{D}}-\bar{R}_{i j \mathrm{WT}}$. The magnitude of the difference is the degree of distortion resulting from the G12D mutation. We present $\Delta \bar{R} i j$ values in the pairwise distances map (Fig. 2), where a positive value indicates that a residue pair moves apart upon G12D mutation, while a negative value indicates that the pair gets 
closer. Then, to identify all residue pairs ( $i j)$ that were significantly distorted by the G12D mutation, we selected the residue pairs that have the greatest (positive and negative) $\Delta \bar{R} i j$ values. We assumed that the residue pairs with $\Delta \bar{R} i j>2.50$ or $<-1.40$ showed the most significant distance changes. For those identified residue pairs, we plot the distribution graphs $W\left(R_{i j}\right)$ of their distances $\left(R_{i j}\right)$ during the simulations of K-Ras ${ }^{\mathrm{WT}}$ and K-Ras ${ }^{\mathrm{G} 12 \mathrm{D}}$ (Figs 3-5, S1, S4-S10; for details see Supplementary Methods).

Then, to quantify the changes in local volumes upon G12D mutation, for each residue $i$ we calculated the average of all $\Delta \bar{R}_{i j}$ values based on the formula $\Delta \bar{R} i=\sum_{j} \Delta \bar{R} i j / N_{n}$, where $N_{n}$ is the number of residues $j$ in the second coordination shell of residue $i$. In detail, for a residue $i$, at the center of a volume $\mathrm{V}$ with a radius of $9.1 \AA$ (the second coordination shell) and we defined the residues $j$ within this volume $\mathrm{V}$ as the neighbors of residue $i$. Then, we calculated the total change in the distance between residue $i$ and its neighbors, $\sum_{j} \Delta \bar{R} i j$, and divided it by the number of neighbors $\sum_{j} \Delta \bar{R} i j / N_{n}$. The resulting $\Delta \bar{R} i$ value is a measure of the change in volume around residue $i$ due to $\mathrm{G} 12 \mathrm{D}$ mutation.

Residue pair correlation calculations. To investigate the coupled motions of residue pairs in protein dynamics, we calculated the correlation coefficients between their fluctuations $\left(C_{i j}\right)$. A correlation coefficient value of a residue pair ranges from -1 to 1 , where for residue pair fluctuations that are not coupled $C i j=0$; perfectly positively correlated $\mathrm{C} i j=1$, and perfectly negatively correlated $\mathrm{C} i j=-1$. We calculated the correlation coefficients as described in our previous study ${ }^{60}$, which we summarize in detail in Supplementary Methods.

The datasets generated and/or analysed during the current study are available from the corresponding authors upon request.

\section{References}

1. Stephen, A. G., Esposito, D., Bagni, R. K. \& McCormick, F. Dragging ras back in the ring. Cancer Cell 25, 272-281, https://doi. org/10.1016/j.ccr.2014.02.017 (2014).

2. Forbes, S. A. et al. COSMIC: exploring the world's knowledge of somatic mutations in human cancer. Nucleic Acids Res 43, D805-811, https://doi.org/10.1093/nar/gku1075 (2015).

3. Prior, I. A., Lewis, P. D. \& Mattos, C. A comprehensive survey of Ras mutations in cancer. Cancer Res 72, 2457-2467, https://doi. org/10.1158/0008-5472.CAN-11-2612 (2012).

4. Pao, W. et al. KRAS mutations and primary resistance of lung adenocarcinomas to gefitinib or erlotinib. Plos Med 2, 57-61, https:// doi.org/10.1371/journal.pmed.0020017 (2005).

5. Amado, R. G. et al. Wild-type KRAS is required for panitumumab efficacy in patients with metastatic colorectal cancer. J Clin Oncol 26, 1626-1634, https://doi.org/10.1200/JCO.2007.14.7116 (2008).

6. Lievre, A. et al. KRAS mutation status is predictive of response to cetuximab therapy in colorectal cancer. Ann Oncol 17, 42-42 (2006).

7. Herrmann, C. \& Nassar, N. Ras and its effectors. Prog Biophys Mol Biol 66, 1-41 (1996).

8. Milburn, M. V. et al. Molecular Switch for Signal Transduction- Structural Differences between Active and Inactive Forms of Protooncogenic Ras Proteins. Science 247, 939-945, https://doi.org/10.1126/science.2406906 (1990).

9. Vetter, I. R. \& Wittinghofer, A. Signal transduction - The guanine nucleotide-binding switch in three dimensions. Science 294, 1299-1304, https://doi.org/10.1126/science.1062023 (2001).

10. Lu, S. et al. Ras Conformational Ensembles, Allostery, and Signaling. Chem Rev 116, 6607-6665, https://doi.org/10.1021/acs. chemrev.5b00542 (2016).

11. Glennon, T. M., Villa, J. \& Warshel, A. How does GAP catalyze the GTPase reaction of Ras?: A computer simulation study. Biochemistry 39, 9641-9651, https://doi.org/10.1021/bi000640e (2000).

12. Scheffzek, K. et al. The Ras-RasGAP complex: Structural basis for GTPase activation and its loss in oncogenic Ras mutants. Science 277, 333-338, https://doi.org/10.1126/science.277.5324.333 (1997).

13. Schubbert, S., Shannon, K. \& Bollag, G. Hyperactive Ras in developmental disorders and cancer. Nat Rev Cancer 7, 295-308, https:// doi.org/10.1038/nrc2109 (2007)

14. Smith, M. J., Neel, B. G. \& Ikura, M. NMR-based functional profiling of RASopathies and oncogenic RAS mutations. Proc Natl Acad Sci USA 110, 4574-4579, https://doi.org/10.1073/pnas.1218173110 (2013).

15. Adjei, A. A. Blocking oncogenic Ras signaling for cancer therapy. J Natl Cancer Inst 93, 1062-1074 (2001).

16. McCormick, F. K-Ras protein as a drug target. J Mol Med (Berl) 94, 253-258, https://doi.org/10.1007/s00109-016-1382-7 (2016).

17. Lu, S., Jang, H., Gu, S., Zhang, J. \& Nussinov, R. Drugging Ras GTPase: a comprehensive mechanistic and signaling structural view. Chem Soc Rev 45, 4929-4952, https://doi.org/10.1039/c5cs00911a (2016).

18. Ostrem, J. M. \& Shokat, K. M. Direct small-molecule inhibitors of KRAS: from structural insights to mechanism-based design. Nat Rev Drug Discov, https://doi.org/10.1038/nrd.2016.139 (2016).

19. Marcus, K. \& Mattos, C. Direct Attack on RAS: Intramolecular Communication and Mutation-Specific Effects. Clin. Cancer Res. 21, 1810-1818, https://doi.org/10.1158/1078-0432.ccr-14-2148 (2015).

20. Malumbres, M. \& Barbacid, M. RAS oncogenes: the first 30 years. Nat Rev Cancer 3, 459-465, https://doi.org/10.1038/nrc1097 (2003).

21. Ito, Y. et al. Regional polysterism in the GTP-bound form of the human c-Ha-Ras protein. Biochemistry 36, 9109-9119, https://doi. org/10.1021/bi970296u (1997).

22. O'Connor, C. \& Kovrigin, E. L. Global conformational dynamics in ras. Biochemistry 47, 10244-10246, https://doi.org/10.1021/ bi801076c (2008).

23. Kearney, B. M., Johnson, C. W., Roberts, D. M., Swartz, P. \& Mattos, C. DRoP: a water analysis program identifies Ras-GTP-specific pathway of communication between membrane-interacting regions and the active site. J Mol Biol 426, 611-629, https://doi. org/10.1016/j.jmb.2013.10.036 (2014).

24. Prakash, P., Zhou, Y., Liang, H., Hancock, J. F. \& Gorfe, A. A. Oncogenic K-Ras Binds to an Anionic Membrane in Two Distinct Orientations: A Molecular Dynamics Analysis. Biophysical Journal 110, 1125-1138, https://doi.org/10.1016/j.bpj.2016.01.019 (2016).

25. Lu, S. Y., Jang, H., Nussinov, R. \& Zhang, J. The Structural Basis of Oncogenic Mutations G12, G13 and Q61 in Small GTPase K-Ras4B. Sci Rep-Uk 6, https://doi.org/10.1038/srep21949 (2016).

26. Prakash, P., Hancock, J. F. \& Gorfe, A. A. Binding hotspots on K-ras: consensus ligand binding sites and other reactive regions from probe-based molecular dynamics analysis. Proteins 83, 898-909, https://doi.org/10.1002/prot.24786 (2015).

27. Chen, C. C. et al. Computational analysis of KRAS mutations: implications for different effects on the KRAS p.G12D and p.G13D mutations. PLoS One 8, e55793, https://doi.org/10.1371/journal.pone.0055793 (2013). 
28. Taveras, A. G. et al. Ras oncoprotein inhibitors: the discovery of potent, ras nucleotide exchange inhibitors and the structural determination of a drug-protein complex. Bioorg Med Chem 5, 125-133 (1997).

29. Ostrem, J. M., Peters, U., Sos, M. L., Wells, J. A. \& Shokat, K. M. K-Ras(G12C) inhibitors allosterically control GTP affinity and effector interactions. Nature 503, 548-551, https://doi.org/10.1038/nature12796 (2013).

30. Lim, S. M. et al. Therapeutic targeting of oncogenic K-Ras by a covalent catalytic site inhibitor. Angew Chem Int Ed Engl 53, 199-204, https://doi.org/10.1002/anie.201307387 (2014).

31. Lito, P., Solomon, M., Li, L. S., Hansen, R. \& Rosen, N. Allele-specific inhibitors inactivate mutant KRAS G12C by a trapping mechanism. Science 351, 604-608, https://doi.org/10.1126/science.aad6204 (2016).

32. Baussand, J. \& Kleinjung, J. Specific Conformational States of Ras GTPase upon Effector Binding. J Chem Theory Comput 9, 738-749, https://doi.org/10.1021/ct3007265 (2013).

33. Scarabelli, G. \& Grant, B. J. Kinesin-5 allosteric inhibitors uncouple the dynamics of nucleotide, microtubule, and neck-linker binding sites. Biophys J 107, 2204-2213, https://doi.org/10.1016/j.bpj.2014.09.019 (2014).

34. Kumar, A., Glembo, T. J. \& Ozkan, S. B. The Role of Conformational Dynamics and Allostery in the Disease Development of Human Ferritin. Biophys J 109, 1273-1281, https://doi.org/10.1016/j.bpj.2015.06.060 (2015).

35. Alred, E. J., Scheele, E. G., Berhanu, W. M. \& Hansmann, U. H. Stability of Iowa mutant and wild type Abeta-peptide aggregates. J Chem Phys 141, 175101, https://doi.org/10.1063/1.4900892 (2014).

36. Gkeka, P. et al. Investigating the structure and dynamics of the PIK3CA wild-type and H1047R oncogenic mutant. PLoS Comput Biol 10, e1003895, https://doi.org/10.1371/journal.pcbi.1003895 (2014).

37. Lu, S. et al. The Mechanism of ATP-Dependent Allosteric Protection of Akt Kinase Phosphorylation. Structure 23, 1725-1734, https://doi.org/10.1016/j.str.2015.06.027 (2015).

38. Blacklock, K. \& Verkhivker, G. M. Computational modeling of allosteric regulation in the hsp90 chaperones: a statistical ensemble analysis of protein structure networks and allosteric communications. PLoS Comput Biol 10, e1003679, https://doi.org/10.1371/ journal.pcbi.1003679 (2014).

39. Sun, H. et al. Revealing the favorable dissociation pathway of type II kinase inhibitors via enhanced sampling simulations and twoend-state calculations. Sci Rep 5, 8457, https://doi.org/10.1038/srep08457 (2015).

40. Sliwoski, G., Kothiwale, S., Meiler, J. \& Lowe, E. W. Jr. Computational methods in drug discovery. Pharmacol Rev 66, 334-395, https://doi.org/10.1124/pr.112.007336 (2014).

41. Stank, A., Kokh, D. B., Fuller, J. C. \& Wade, R. C. Protein Binding Pocket Dynamics. Acc Chem Res 49, 809-815, https://doi. org/10.1021/acs.accounts.5b00516 (2016).

42. Borhani, D. W. \& Shaw, D. E. The future of molecular dynamics simulations in drug discovery. J Comput Aided Mol Des 26, 15-26, https://doi.org/10.1007/s10822-011-9517-y (2012).

43. Durrant, J. D. \& McCammon, J. A. Molecular dynamics simulations and drug discovery. BMC Biol 9, 71, https://doi. org/10.1186/1741-7007-9-71 (2011).

44. Bahar, I., Atilgan, A. R. \& Erman, B. Direct evaluation of thermal fluctuations in proteins using a single-parameter harmonic potential. Fold Des 2, 173-181, https://doi.org/10.1016/S1359-0278(97)00024-2 (1997).

45. Haliloglu, T., Bahar, I. \& Erman, B. Gaussian dynamics of folded proteins. Physical Review Letters 79, 3090-3093, https://doi. org/10.1103/PhysRevLett.79.3090 (1997).

46. Bahar, I., Atilgan, A. R., Demirel, M. C. \& Erman, B. Vibrational dynamics of folded proteins: Significance of slow and fast motions in relation to function and stability. Physical Review Letters 80,2733-2736, https://doi.org/10.1103/PhysRevLett.80.2733 (1998).

47. Atilgan, A. R., Akan, P. \& Baysal, C. Small-world communication of residues and significance for protein dynamics. Biophys J 86, 85-91, https://doi.org/10.1016/S0006-3495(04)74086-2 (2004).

48. Downward, J. Targeting ras signalling pathways in cancer therapy. Nature Reviews Cancer 3, 11-22, https://doi.org/10.1038/nrc969 (2003).

49. Lu, S. et al. GTP Binding and Oncogenic Mutations May Attenuate Hypervariable Region (HVR)-Catalytic Domain Interactions in Small GTPase K-Ras4B, Exposing the Effector Binding Site. J Biol Chem 290, 28887-28900, https://doi.org/10.1074/jbc. M115.664755 (2015).

50. Johnson, C. W. et al. The small GTPases K-Ras, N-Ras, and H-Ras have distinct biochemical properties determined by allosteric effects. J Biol Chem 292, 12981-12993, https://doi.org/10.1074/jbc.M117.778886 (2017).

51. Parker, J. A., Volmar, A. Y., Pavlopoulos, S. \& Mattos, C. K-Ras Populates Conformational States Differently from Its Isoform H-Ras and Oncogenic Mutant K-RasG12D. Structure 26, 810-820 e814, https://doi.org/10.1016/j.str.2018.03.018 (2018).

52. Buhrman, G., Holzapfel, G., Fetics, S. \& Mattos, C. Allosteric modulation of Ras positions Q61 for a direct role in catalysis. Proc Natl Acad Sci USA 107, 4931-4936, https://doi.org/10.1073/pnas.0912226107 (2010).

53. Buhrman, G., Kumar, V. S., Cirit, M., Haugh, J. M. \& Mattos, C. Allosteric modulation of Ras-GTP is linked to signal transduction through RAF kinase. J Biol Chem 286, 3323-3331, https://doi.org/10.1074/jbc.M110.193854 (2011).

54. Buhrman, G. et al. Analysis of binding site hot spots on the surface of Ras GTPase. J Mol Biol 413, 773-789, https://doi.org/10.1016/j. jmb.2011.09.011 (2011).

55. Johnson, C. W. \& Mattos, C. The allosteric switch and conformational states in Ras GTPase affected by small molecules. Enzymes 33(Pt A), 41-67, https://doi.org/10.1016/B978-0-12-416749-0.00003-8 (2013).

56. Gorfe, A. A., Grant, B. J. \& McCammon, J. A. Mapping the nucleotide and isoform-dependent structural and dynamical features of Ras proteins. Structure 16, 885-896, https://doi.org/10.1016/j.str.2008.03.009 (2008).

57. Prakash, P., Sayyed-Ahmad, A. \& Gorfe, A. A. The role of conserved waters in conformational transitions of Q61H K-ras. PLoS Comput Biol 8, e1002394, https://doi.org/10.1371/journal.pcbi.1002394 (2012).

58. Sayyed-Ahmad, A., Prakash, P. \& Gorfe, A. A. Distinct dynamics and interaction patterns in H- and K-Ras oncogenic P-loop mutants. Proteins 85, 1618-1632, https://doi.org/10.1002/prot.25317 (2017).

59. Pantsar, T. et al. Assessment of mutation probabilities of KRAS G12 missense mutants and their long-timescale dynamics by atomistic molecular simulations and Markov state modeling. PLoS Comput Biol 14, e1006458, https://doi.org/10.1371/journal. pcbi.1006458 (2018).

60. Vatansever, S., Gumus, Z. H. \& Erman, B. Intrinsic K-Ras dynamics: A novel molecular dynamics data analysis method shows causality between residue pair motions. Sci Rep 6, 37012, https://doi.org/10.1038/srep37012 (2016).

61. Discovery Studio Modeling Environment v. Release 4.5 (San Diego: Dassault Systèmes, 2015).

62. Phillips, J. C. et al. Scalable molecular dynamics with NAMD. Journal of Computational Chemistry 26, 1781-1802, https://doi. org/10.1002/jcc.20289 (2005).

63. Hornak, V. et al. Comparison of multiple amber force fields and development of improved protein backbone parameters. ProteinsStructure Function and Bioinformatics 65, 712-725, https://doi.org/10.1002/prot.21123 (2006).

64. Wang, J. M., Wolf, R. M., Caldwell, J. W., Kollman, P. A. \& Case, D. A. Development and testing of a general amber force field. Journal of Computational Chemistry 25, 1157-1174, https://doi.org/10.1002/jcc.20035 (2004).

65. Humphrey, W., Dalke, A. \& Schulten, K. VMD: Visual molecular dynamics. J Mol Graph Model 14, 33-38, https://doi. org/10.1016/0263-7855(96)00018-5 (1996).

66. Woodcock, L. V. Entropy difference between the face-centred cubic and hexagonal close-packed crystal structures. Nature 385, 141-143, https://doi.org/10.1038/385141a0 (1997). 


\section{Acknowledgements}

Z.H.G. acknowledges funding from the LUNGevity Foundation and start-up funds from the Icahn Institute at Icahn School of Medicine at Mount Sinai. We would also like to thank Dr. Roman Osman for valuable comments on the manuscript and Dr. Myvizhi Esai Selvan for help with one of the figures.

\section{Author Contributions}

S.V. conducted the molecular dynamics simulations and data analyses, prepared the figures and evaluated results. Z.H.G. and B.E. contributed to study design and evaluation of the results. Z.H.G. and B.E. contributed oversight to the study. All authors have contributed to manuscript drafting and agree to the submitted manuscript.

\section{Additional Information}

Supplementary information accompanies this paper at https://doi.org/10.1038/s41598-019-48029-z.

Competing Interests: The authors declare no competing interests.

Publisher's note: Springer Nature remains neutral with regard to jurisdictional claims in published maps and institutional affiliations.

(c) (i) Open Access This article is licensed under a Creative Commons Attribution 4.0 International License, which permits use, sharing, adaptation, distribution and reproduction in any medium or format, as long as you give appropriate credit to the original author(s) and the source, provide a link to the Creative Commons license, and indicate if changes were made. The images or other third party material in this article are included in the article's Creative Commons license, unless indicated otherwise in a credit line to the material. If material is not included in the article's Creative Commons license and your intended use is not permitted by statutory regulation or exceeds the permitted use, you will need to obtain permission directly from the copyright holder. To view a copy of this license, visit http://creativecommons.org/licenses/by/4.0/.

(C) The Author(s) 2019 